\title{
Does the Swiss School of Public Health exist?
}

\author{
Nino Künzli $\cdot$ Luca Crivelli $\cdot$ Dominique Sprumont $\cdot$ Sandra Nocera
}

Received: 17 October 2015/ Accepted: 18 October 2015/Published online: 3 November 2015

(C) Swiss School of Public Health (SSPH+) 2015

"Switzerland does not exist" or "La Suisse n'existe pas" was the statement the French artist Ben Vautier featured in the Swiss Pavilion at the World Exposition 1992 in Seville. It provoked an outcry in Switzerland, in particular in the political arenas of parliaments and the media. The Swiss School of Public Health (SSPH +) - proud owner of the International Journal of Public Health-celebrated the 10th anniversary in 2015. Why would the SSPH+ Directorate refer to Ben Vautier's provocation to ask whether SSPH+ does exist?

As SSPH+ moves into the second decade it will face the challenges of puberty and the years as a teenager. These are times of transitions, changes and reflections too. Table 1 summarizes the major achievements and highlights of the first 10 years of SSPH+. These successes, achieved under

This Editorial was published on occasion of the 10th anniversary of the Swiss School of Public Health.

N. Künzli $\cdot$ L. Crivelli $\cdot$ D. Sprumont $\cdot$ S. Nocera

Swiss School of Public Health (SSPH+), Zurich, Switzerland

N. Künzli $(\bowtie)$

Swiss Tropical and Public Health Institute, Socinstrasse 57, 4002 Basel, Switzerland

e-mail: Nino.Kuenzli@unibas.ch

\section{N. Künzli}

University of Basel, Basel, Switzerland

D. Sprumont

University of Neuchâtel, Neuchâtel, Switzerland

L. Crivelli

Università della Svizzera italiana, Lugano, Switzerland

L. Crivelli

University of Applied Sciences and Arts of Southern

Switzerland, Manno, Switzerland the leadership of the past Directors were also the result of the solid funding structure. SSPH+, initiated in July 2005 through funds from the Swiss University Conference (SUC), was transformed into a Foundation as of 2008. At that time, SSPH+ expanded its network from six to eight Swiss universities, including all universities with major academic institutions of public health, namely Basel, Bern, Geneva, Lausanne, Lucerne, Lugano, Neuchâtel and Zurich. The SUC support for SSPH+ was reserved for the first 12 years to then adopt a new financing model. Indeed, as of 2017, the SUC funds-in the past some 1-3 Mio CHF per year-will be replaced by a model where all the universities that carry SSPH+ would become its direct funding bodies.

This visionary model for a School of Public Health being a network of all academic public health constituencies rather than a classic central "school" is intriguing for a small country like Switzerland. The model gives a promising structure to strengthen public health in a country where excessively federalistic structures resulted so far in rather weak public health systems and infrastructurescomparable to the situation in Germany where an expert panel properly identified the need for strengthening the field (German National Academy of Sciences Leopoldina et al. 2015).

Whether we will accomplish this goal in the next decade will depend on the future willingness to collaborate, which in turn will also depend on sustainable funds. At this stage, the foundation has neither been set on balanced nor stable grounds for its upcoming time as a teenager. As the budget will be lower than in the past and commitments of the eight university partners unequal (contributions of each university range between 5 and $60 \%$ of the future budget) one primary strategic goal of the Directorate will be to strengthen the financial pillars of SSPH+. 
Table 1 Major achievements and activities of the network of the Swiss School of Public Health $(\mathrm{SSPH}+)$ during the first 10 years, 2005-2015

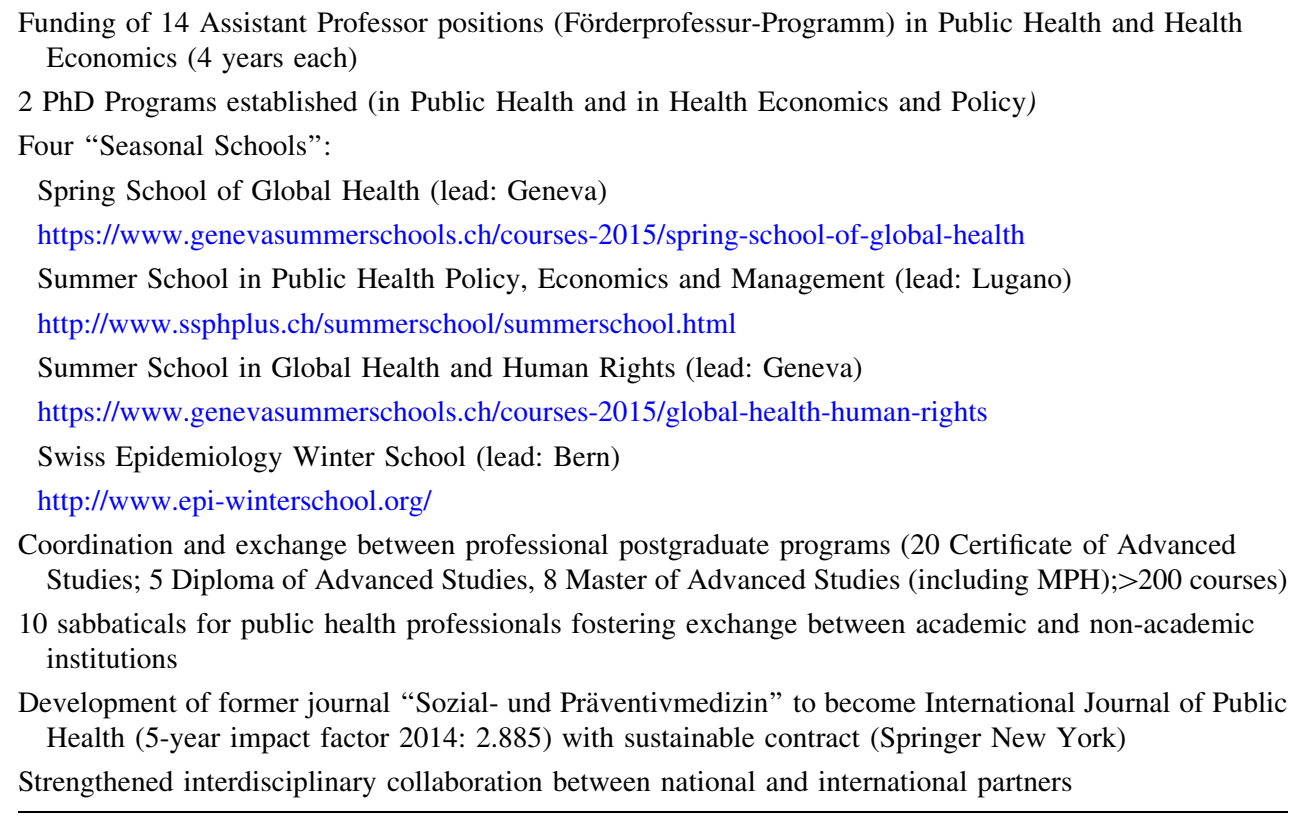

One of the missions of SSPH+ is to promote and maintain a skilled public health workforce at local, national and international level through high quality education and training. With the rapid changes in the graduate and postgraduate landscape of public health careers, there is a need for leadership in shaping and qualifying the tracks in public health education. We must accomplish the goal that not only the current $31 \%$ but indeed $100 \%$ of the Swiss public health workforce will have a formal training in public health (Paccaud et al. 2013). This objective is ambitious but in line with the Federal Council's strategy for health in Switzerland (Gesundheit2020/Santé2020/Salute2020) which sets priorities of public health in Switzerland for the coming years (FDHA 2013). In fact, the strategic plan makes a visionary statement in its objective 3.3.: "More attention needs to be paid to public health training because the demand for these professionals in public administration and both profit-making and nonprofit organizations is set to increase constantly". We fully agree and in fact the needs in Switzerland are in line with those identified for Europe at large (Bjegovic-Mikanovic et al. 2013). SSPH+ provides the perfect network to contribute to those needs of the Federal Office of Public Health. Unfortunately, the cited objective does not appear in the prioritization as outlined in the ten next steps of the current version of the strategic plan. However, a strategy is a process that undergoes continuous refinement and we are convinced the Federal Council will specify and also prioritize commitments necessary toward the goal of guaranteeing a sufficient workforce of public health professionals.
The time has also come to re-discuss the current career tracks in public health in light of the many changes that shake all educational landscapes. SSPH+ has to discuss the current building blocks of the academic public health careers in Switzerland. We have to critically review whether a public health bachelor degree-not yet existing in Switzerland-will need to be offered in the future. How the fundamental changes in educational tracks may require adaptations in our MPH programs is another subject of relevance. These postgraduate programs are challenged not only by the increasingly harsh economic environment but indeed the many alternative tracks where major elements of an MPH program can now be acquired through otherpossibly less expensive_paths, including online courses or new pre-graduate master programs offered by a few Swiss Universities or Universities of Applied Sciences. Opportunities that come along with these challenges need to be identified not only nationally but through our international educational collaborations and networks as members of ASPHER (http://aspher.org/), tropEd (http://www.troped. org/) and other relevant and successful constituencies in the field of public health education.

To strengthen the link between research and practice and to influence research, training and policy making remains another mission of SSPH+. In that regard, the SSPH+ network is dedicated to support a national population based large-scale cohort study and biobank. Such "mega-cohorts" are not only instrumental for public health but indeed a fundamentally relevant pillar of "personalized health" strategies which must fully integrate public health perspectives. SSPH+ unifies the expertise needed to 
generate the data platform linking life and public health sciences to tackle, in a transdisciplinary manner, the challenges of health, health systems, and diseases in an integrated and equitable manner. Last but not least, with the broad international networks that also characterize $\mathrm{SSPH}+$, we provide also the tools and expertise to compare threats, opportunities and solutions across systems and cultures.

In sum, SSPH+ is looking forward to tackle new options and opportunities relevant for public health academia. In pursuing our goals and thanks to the commitment of all our fellow partners, we are convinced that the answer to the provocative question is "yes". SSPH+ does and will exist-and we are looking forward to celebrate the 20th birthday in 2025 !

\section{References}

Bjegovic-Mikanovic V, Vukovic D, Otok R, Czabanowska K, Laaser $\mathrm{U}$ (2013) Education and training of public health professionals in the European Region: variation and convergence. Int J Public Health 58(6):801-810
FDHA Federal Department of Home Affairs (2013) The Federal Council's health-policy priorities. Bern: Swiss Confederation, Report. http://www.bag.admin.ch/gesundheit2020/index.html? lang=en. Accessed 14 Oct 2015

German National Academy of Sciences Leopoldina, acatech, National Academy of Science and Engineering, Union of the German Academies of Sciences and Humanities (2015) Public Health in Germany-structures, developments and global challenges. Halle (Saale). 76 pages, ISBN 978-3-8047-3346-6. 14 Oct 2015 http://www.leopoldina.org/nc/en/publications/detailview/ ?publication[publication] $=670 \& \mathrm{cHash}=37071954 \mathrm{e} 7942 \mathrm{f} 41 \mathrm{af} 469$ bad12ed5b0d. Accessed 29 Oct 2015

Paccaud F, Weihofen A, Frank M (2013) Public Health Workforce in Switzerland: are public health workers lacking? Int J Public Health 58(6):799-800 